\title{
Differential virial theorem in relation to a sum rule for the exchange- correlation force in density-functional theory
}

\author{
A. Holas ${ }^{a)}$ \\ Institute of Physical Chemistry, Polish Academy of Sciences, 44/52 Kasprzaka, 01-224 Warsaw, Poland \\ N. H. March \\ Donastia International Physics Center, E-20018 San Sebastián, Spain and Oxford University, \\ Oxford OX1 3QR, England \\ Angel Rubio \\ Institut für Theoretische Physik, Freie Universität Berlin, Arnimallee 14, D-14 195 Berlin, Germany, \\ Departamento de Física de Materiales, Facultad de Ciencias Químicas, Universidad del País Vasco/Euskal \\ Herriko Unibertsitatea (UPV/EHU) and Centro Mixto Consejo Superior de Investigaciones Científicas- \\ Universidad del País Vasco/Euskal Herriko Unibertsitatea (CSIC-UPV/EHU), E-20018 San Sebastián, Spain, \\ and Donastia International Physics Center, E-20018 San Sebastián, Spain
}

(Received 7 September 2005; accepted 15 September 2005; published online 11 November 2005)

Holas and March [Phys. Rev. A. 51, 2040 (1995)] gave a formally exact theory for the exchange-correlation (xc) force $\boldsymbol{F}_{\mathrm{xc}}(\boldsymbol{r})=-\boldsymbol{\nabla} v_{\mathrm{xc}}(\boldsymbol{r})$ associated with the xc potential $v_{\mathrm{xc}}(\boldsymbol{r})$ of the density-functional theory in terms of low-order density matrices. This is shown in the present study to lead, rather directly, to the determination of a sum rule $\left\langle n \boldsymbol{F}_{\mathrm{xc}}\right\rangle=0$ relating the xc force with the ground-state density $n(\boldsymbol{r})$. Some connection is also made with an earlier result relating to the external potential by Levy and Perdew [Phys. Rev. A. 32, 2010 (1985)] and with the quite recent study of Joubert [J. Chem. Phys. 119, 1916 (2003)] relating to the separation of the exchange and correlation contributions. (C) 2005 American Institute of Physics. [DOI: 10.1063/1.2114848]

\section{BACKGROUND AND OUTLINE}

While density-functional theory (DFT) continues to be widely applied to a variety of problems embracing both chemical physics and biology, evidence is building up that existing approximate functionals are in urgent need of refinement. One very recent example of this is the study by Wanko et al. ${ }^{1}$ of absorption properties of retinal proteins, for which presently available functionals lead to a disagreement with experiment.

In the search for such refined exchange-correlation (xc) energy functionals, exact results, though generally of integral rather than local form, involving the xc potential $v_{\mathrm{xc}}(\boldsymbol{r})$, can be expected to play an increasingly important role in testing the quantitative virtues of proposed refinements. The present study lies in this area but focuses on the xc force $\boldsymbol{F}_{\mathrm{xc}}(\boldsymbol{r})$ defined by

$$
\boldsymbol{F}_{\mathrm{xc}}(\boldsymbol{r})=-\boldsymbol{\nabla} v_{\mathrm{xc}}(\boldsymbol{r}) \text {. }
$$

This quantity was treated exactly by Holas and $\mathrm{March}^{2}$ in terms of low-order density matrices (DMs) via the so-called differential form of the virial theorem. It should be stressed that in the present paper we will deal exclusively with finite systems, that is, with molecules and clusters.

With this background, the outline of the present article is as follows. In Sec. II, after recalling the March-Young ${ }^{3}$ result as a background, we first set out the Holas-March ${ }^{2}$ result based on the differential virial theorem. As a consequence, we are led to Eq. (2.7) for the average force $-\nabla v_{\text {ext }}(\boldsymbol{r})$ asso-

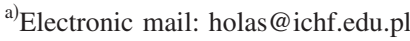

ciated with the external potential. This involves the vector field $z(\boldsymbol{r})$ of the kinetic origin (embracing the kinetic correlation energy) as well as the electron-electron interaction term. A connection is made with an early result of Levy and $\mathrm{Perdew}^{4}$ for this average force. Throughout Sec. II, we work with the exact exchange-correlation potential without needing any separation into exchange and correlation contributions. In Sec. III, however, we perform such separation and make connections with an earlier study of Joubert. ${ }^{5}$ Section IV concludes with a brief summary of the main findings of the present article.

\section{EXACT SUM RULES OBTAINED VIA THE DIFFERENTIAL VIRIAL THEOREM}

\section{A. Sum rules in a one-dimensional noninteracting system}

As a background to the differential virial theorem in this context, we start from the one-dimensional form derived by March and Young. ${ }^{3}$ They introduced the kinetic energy per unit length $t(x)$ and considered an arbitrary number of noninteracting fermions moving in a common one-dimensional potential field $v(x)$. By appealing to the equation of motion for the Dirac density matrix [single particle $(s)$ and therefore idempotent $] \rho^{s}\left(x_{1} ; x_{2}\right)$, expanding it near the diagonal and utilizing the fermion density $n(x)=\rho^{s}(x ; x)$, March and Young found 


$$
\frac{d t(x)}{d x}=-\frac{1}{2} n(x) \frac{d v(x)}{d x}+\frac{1}{8} \frac{d^{3} n(x)}{d x^{3}}
$$

[rewritten here using an alternative definition of the kineticenergy density as $t(x)=\frac{1}{2} \partial^{2} \rho^{s}\left(x^{\prime} ; x^{\prime \prime}\right) /\left.\partial x^{\prime} \partial x^{\prime \prime}\right|_{x^{\prime}=x^{\prime \prime}=x}$; atomic units are used throughout the paper]. Forming the virial of Clausius $\boldsymbol{r} \cdot \boldsymbol{F}(\boldsymbol{r})$ which in one dimension is evidently $x F(x)$ $=-x d v(x) / d x$, they stressed that Eq. (2.1), multiplied by $2 x$ and integrated, gave back the usual integral virial theorem

$$
2 T=-\int_{-\infty}^{\infty} d x n(x) x F(x)
$$

where $T=\int_{-\infty}^{\infty} d x t(x)$. Thus Eq. (2.1) was termed the differential form of the virial theorem. This equation is readily rewritten as a "force-balance" equation, which then reads

$$
F(x)=\frac{d v(x)}{d x}=\left[2 \frac{d t(x)}{d x}-\frac{1}{4} \frac{d^{3} n(x)}{d x^{3}}\right] \frac{1}{n(x)} .
$$

The achievement of Holas and March ${ }^{2}$ was to effect the exact generalization of the independent-fermion result (2.3) to three dimensions and, of course, to include also a full account of electron-electron interactions.

\section{B. Sum rule for the external force in a three-dimensional interacting system}

Let us start from the Holas-March ${ }^{2}$ result for the external force stemming from the differential virial theorem for the interacting many-electron system, which reads [their Eq. (2.16)]

$$
\begin{aligned}
\boldsymbol{F}_{\mathrm{ext}}(\boldsymbol{r})= & -\boldsymbol{\nabla} v_{\mathrm{ext}}(\boldsymbol{r})=\left\{z(\boldsymbol{r} ;[\rho])-\frac{1}{4} \boldsymbol{\nabla} \nabla^{2} n(\boldsymbol{r})\right. \\
& \left.+2 \int d^{3} r^{\prime} n_{2}\left(\boldsymbol{r}, \boldsymbol{r}^{\prime}\right) \boldsymbol{\nabla}_{\boldsymbol{r}}\left(\left|\boldsymbol{r}-\boldsymbol{r}^{\prime}\right|^{-1}\right)\right\} / n(\boldsymbol{r}) .
\end{aligned}
$$

Here $n_{2}\left(\boldsymbol{r}, \boldsymbol{r}^{\prime}\right)$ denotes the pair density, which is the diagonal element of the second-order density matrix (2DM). The vector field $z(\boldsymbol{r} ;[\rho])$ is defined solely in terms of the fully interacting first-order density matrix (1DM) $\rho\left(\boldsymbol{r}_{1} ; \boldsymbol{r}_{2}\right)$ via the kinetic-energy density tensor $t_{\alpha \beta}$ as

$$
z_{\alpha}(\boldsymbol{r} ;[\rho])=2 \sum_{\beta} \frac{\partial}{\partial r_{\beta}} t_{\alpha \beta}(\boldsymbol{r} ;[\rho]),
$$

the precise definition of this tensor employed in Ref. 2 being

$$
t_{\alpha \beta}(\boldsymbol{r} ;[\rho])=\frac{1}{4}\left(\frac{\partial^{2}}{\partial r_{\alpha}^{\prime} \partial r_{\beta}^{\prime \prime}}+\frac{\partial^{2}}{\partial r_{\beta}^{\prime} \partial r_{\alpha}^{\prime \prime}}\right) \rho\left(\boldsymbol{r}^{\prime} ; \boldsymbol{r}^{\prime \prime}\right)_{\boldsymbol{r}^{\prime}=r^{\prime \prime}=\boldsymbol{r}} .
$$

Using the notation $\langle f\rangle=\int d^{3} r f(\boldsymbol{r})$, we find from Eq. (2.4) the result

$$
\begin{aligned}
\left\langle n \boldsymbol{F}_{\mathrm{ext}}\right\rangle= & \int d^{3} r z(\boldsymbol{r} ;[\rho])-\frac{1}{4} \int d^{3} r \nabla \nabla^{2} n(\boldsymbol{r}) \\
& +2 \int d^{3} r d^{3} r^{\prime} n_{2}\left(\boldsymbol{r}, \boldsymbol{r}^{\prime}\right) \nabla_{\boldsymbol{r}}\left(\left|\boldsymbol{r}-\boldsymbol{r}^{\prime}\right|^{-1}\right) .
\end{aligned}
$$

\section{Sum rule for the external force in a noninteracting system}

Adapting the result (2.4) to a system of noninteracting fermions moving in a common potential field $v_{s}(\boldsymbol{r})$, we omit the term due to the electron-electron interaction (the integral) and replace the interacting 1DM $\rho\left(\boldsymbol{r}_{1} ; \boldsymbol{r}_{2}\right)$ by its singleparticle counterpart $\rho^{s}\left(\boldsymbol{r}_{1} ; \boldsymbol{r}_{2}\right)$. The external force in this system [Eq. (2.24) in Ref. 2] then reads

$$
\boldsymbol{F}_{s}(\boldsymbol{r})=-\boldsymbol{\nabla} v_{s}(\boldsymbol{r})=\left\{\boldsymbol{z}\left(\boldsymbol{r} ;\left[\rho^{s}\right]\right)-\frac{1}{4} \boldsymbol{\nabla} \nabla^{2} n(\boldsymbol{r})\right\} / n(\boldsymbol{r}) .
$$

This leads to a sum rule analogous to Eq. (2.7)

$$
\left\langle n \boldsymbol{F}_{s}\right\rangle=\int d^{3} r z\left(\boldsymbol{r} ;\left[\rho^{s}\right]\right)-\frac{1}{4} \int d^{3} r \nabla \nabla^{2} n(\boldsymbol{r}) .
$$

\section{Sum rules for forces in the Kohn-Sham system}

Considering now the noninteracting system of Sec. II C to be the Kohn-Sham (KS) system of DFT (see, e.g., Ref. 6) equivalent to the original, interacting system of Sec. II B and, therefore, having the same ground-state density $n(\boldsymbol{r})$, we identify its external potential with the formally exact KS potential

$$
v_{s}(\boldsymbol{r}) \equiv v_{\mathrm{KS}}(\boldsymbol{r})=v_{\mathrm{ext}}(\boldsymbol{r})+v_{\mathrm{esxc}}(\boldsymbol{r}),
$$

where $v_{\mathrm{esxc}}(\boldsymbol{r})$ denotes the electrostatic-plus-exchangecorrelation potential. The difference between Eqs. (2.7) and (2.9) with the partition (2.10) taken into account leads immediately to the sum rule for the esxc force $\boldsymbol{F}_{\text {esxc }}(\boldsymbol{r})$ $=-\nabla v_{\text {esxc }}(\boldsymbol{r})$, namely,

$$
\begin{aligned}
\left\langle n \boldsymbol{F}_{\mathrm{esxc}}\right\rangle= & \int d^{3} r z\left(\boldsymbol{r} ;\left[\rho^{s}-\rho\right]\right) \\
& -2 \int d^{3} r d^{3} r^{\prime} n_{2}\left(\boldsymbol{r}, \boldsymbol{r}^{\prime}\right) \nabla_{\boldsymbol{r}}\left(\left|\boldsymbol{r}-\boldsymbol{r}^{\prime}\right|^{-1}\right) .
\end{aligned}
$$

It can be reduced next to

$$
\left\langle n \boldsymbol{F}_{\text {esxc }}\right\rangle=\mathbf{0} .
$$

The vanishing in Eq. (2.11) of the electron-electron interaction contribution is guaranteed by the symmetry of the integrand: the factor $\nabla_{\boldsymbol{r}}\left(\left|\boldsymbol{r}-\boldsymbol{r}^{\prime}\right|^{-1}\right)=-\left(\boldsymbol{r}-\boldsymbol{r}^{\prime}\right) /\left|\boldsymbol{r}-\boldsymbol{r}^{\prime}\right|^{3}$ is antisymmetric in $\left\{\boldsymbol{r}, \boldsymbol{r}^{\prime}\right\}$, while the remaining factor is symmetric. The vanishing of the kinetic-energy contribution (depending on $\rho^{s}-\rho$ ) is due to the asymptotic large- $r$ exponential decay of 1DMs: the long-range behavior of each natural orbital of $\rho$ and of the most extended occupied KS orbital of $\rho^{s}$ is proportional to $\exp \left[-\left(2 I_{\min }\right)^{-1 / 2} r\right]$, where $I_{\min }$ denotes the ionization potential of the system (see, e.g., Refs. 7 and 6). This applies to finite systems only which are considered in the present paper.

The same arguments lead to the vanishing of all terms in both Eqs. (2.7) and (2.9), so the identities

$$
\left\langle n \boldsymbol{F}_{\text {ext }}\right\rangle=0, \quad\left\langle n \boldsymbol{F}_{s}\right\rangle=\mathbf{0}
$$

follow. They reveal the homogeneity of the space as shown by Levy and Perdew. ${ }^{4}$ 
The identities (2.7) and (2.9) (representing relations between external potentials, interaction potential, and DMs) depend linearly on DMs derived from any eigenfunction of an interacting system $\left(n, \rho, n_{2}\right)$ and of a noninteracting one $\left(n, \rho^{s}\right)$, respectively. Therefore these relations remain true if the pure-state DMs are replaced by the corresponding ensemble DMs. While the DMs generated from the groundstate eigenfunctions are used for application in the basic DFT, those generated from the excited-state eigenfunctions can be applied in the corresponding DFT extension (see, e.g., Levy and $\mathrm{Nagy}^{8}$ ). All above-mentioned remarks concern also Eq. (2.11), which represents the difference of Eqs. (2.7) and (2.9).

Finally, by taking $v_{\mathrm{xc}}(\boldsymbol{r})=v_{\mathrm{esxc}}(\boldsymbol{r})-v_{\mathrm{es}}(\boldsymbol{r})$, the sum rule for the $\mathrm{xc}$ force

$$
\left\langle n \boldsymbol{F}_{\mathrm{xc}}\right\rangle=\mathbf{0}
$$

can be obtained from Eq. (2.12) by subtracting the contribution due to the electrostatic force $\boldsymbol{F}_{\mathrm{es}}(\boldsymbol{r})=-\boldsymbol{\nabla} v_{\mathrm{es}}(\boldsymbol{r})$. Here, the electrostatic potential is defined as usually by

$$
v_{\mathrm{es}}(\boldsymbol{r} ;[n])=\int d^{3} r^{\prime} n\left(\boldsymbol{r}^{\prime}\right) /\left|\boldsymbol{r}-\boldsymbol{r}^{\prime}\right| .
$$

The identity, which is applied for obtaining Eq. (2.14),

$$
\left\langle n \boldsymbol{F}_{\mathrm{es}}\right\rangle=-\int d^{3} r d^{3} r^{\prime} n(\boldsymbol{r}) n\left(\boldsymbol{r}^{\prime}\right) \boldsymbol{\nabla}_{\boldsymbol{r}}\left(\left|\boldsymbol{r}-\boldsymbol{r}^{\prime}\right|^{-1}\right)=\mathbf{0},
$$

holds due to the symmetry of the integrand, as discussed just below Eq. (2.12). In the case of the ensemble DFT, the ensemble density $n(\boldsymbol{r})$ is to be used in Eq. (2.15).

\section{SEPARATE SUM RULES FOR THE EXCHANGE AND CORRELATION FORCES}

As shown by Levy and March, ${ }^{9}$ the separate expressions for the exact exchange and correlation forces can be extracted from the Holas-March ${ }^{2}$ result for the esxc force $\boldsymbol{F}_{\text {esxc }}$ through the use of the coupling-constant adiabaticconnection method. ${ }^{10}$ Görling and Levy ${ }^{10}$ proposed to link the interacting system and the equivalent noninteracting $\mathrm{KS}$ system by a family of intermediate systems with the electron-electron interaction $\lambda /\left|\boldsymbol{r}-\boldsymbol{r}^{\prime}\right|$, i.e., scaled by the coupling parameter $\lambda \in[0,1]$; all systems are required to have the same density $n(\boldsymbol{r})$. Properties of the fully interacting system (at $\lambda=1)$ are viewed as derived from the KS system solution $($ at $\lambda=0$ ) with the help of the perturbation theory with respect to $\lambda$. In this approach, the expanded esxc force in the intermediate system

$$
\boldsymbol{F}_{\mathrm{esxc}}^{\lambda}(\boldsymbol{r})=\sum_{l=1}^{\infty} \lambda^{l} \boldsymbol{F}_{\mathrm{esxc}}^{\{l\}}(\boldsymbol{r})
$$

gives the electrostatic-plus-exchange force $\boldsymbol{F}_{\text {esx }}$ as its leading term, and the correlation force $\boldsymbol{F}_{c}$ as the sum of the remaining terms:

$$
\boldsymbol{F}_{\mathrm{esx}}(\boldsymbol{r})=\boldsymbol{F}_{\mathrm{esxc}}^{\{1\}}(\boldsymbol{r}),
$$

$$
\begin{aligned}
& \boldsymbol{F}_{c}(\boldsymbol{r})=\boldsymbol{F}_{\mathrm{esxc}}(\boldsymbol{r})-\boldsymbol{F}_{\mathrm{esx}}(\boldsymbol{r})=\sum_{l=2}^{\infty} \boldsymbol{F}_{c}^{\{l\}}(\boldsymbol{r}), \\
& \forall l \geqslant 2, \quad \boldsymbol{F}_{c}^{\{l\}}(\boldsymbol{r})=\boldsymbol{F}_{\mathrm{esxc}}^{\{l\}}(\boldsymbol{r}) .
\end{aligned}
$$

The sum rule for the esxc force $\boldsymbol{F}_{\text {esxc }}^{\lambda}$ in the intermediate system is given by Eq. (2.11) adapted to this system, i.e., with the DMs $\rho, n_{2}$ replaced by $\rho^{\lambda}, n_{2}^{\lambda}$ and with the interaction potential scaled by $\lambda$ :

$$
\begin{aligned}
\left\langle n \boldsymbol{F}_{\mathrm{esxc}}^{\lambda}\right\rangle= & \int d^{3} r z\left(\boldsymbol{r} ;\left[\rho^{s}-\rho^{\lambda}\right]\right) \\
& -2 \int d^{3} r d^{3} r^{\prime} n_{2}^{\lambda}\left(\boldsymbol{r}, \boldsymbol{r}^{\prime}\right) \boldsymbol{\nabla}_{\boldsymbol{r}}\left(\lambda\left|\boldsymbol{r}-\boldsymbol{r}^{\prime}\right|^{-1}\right) .
\end{aligned}
$$

This again can be simplified to

$$
\left\langle n \boldsymbol{F}_{\text {esxc }}^{\lambda}\right\rangle=0, \quad \forall \lambda \in[\mathbf{0}, 1]
$$

by applying the symmetry argument to the interaction contribution and by noting that the asymptotic exponential decay of natural orbitals of $\rho^{\lambda}$ should be of the same form at any strength $\lambda$ of the interaction because $n^{\lambda}(\boldsymbol{r})=n(\boldsymbol{r})$. The sum rule (3.4) expanded according to Eq. (3.1) results in

$$
\left\langle n \boldsymbol{F}_{\mathrm{esxc}}^{\{l\}}\right\rangle=\mathbf{0} \text { for } l=1,2, \ldots .
$$

The following sum rules follow from Eq. (3.5) and the assignments (3.2a), (3.2b), and (3.2c):

$$
\begin{aligned}
& \left\langle n \boldsymbol{F}_{\text {esx }}\right\rangle=\mathbf{0}, \\
& \left\langle n \boldsymbol{F}_{c}\right\rangle=\mathbf{0}, \\
& \left\langle n \boldsymbol{F}_{c}^{\{l\}}\right\rangle=\mathbf{0}, \quad \text { for } l=2,3, \ldots .
\end{aligned}
$$

The results (3.6a), (3.6b), and (3.6c) are identical with the equations derived by Joubert ${ }^{5}$ with the help of different methods.

Finally, by taking $\boldsymbol{F}_{x}=\boldsymbol{F}_{\text {esx }}-\boldsymbol{F}_{\text {es }}$ and using the results (2.16) and (3.6a), the separate sum rule for the exchange force is obtained:

$$
\left\langle n \boldsymbol{F}_{\mathrm{X}}\right\rangle=\mathbf{0} .
$$

\section{DISCUSSION AND SUMMARY}

Everything calculated in the present study in Sec. II stems from the differential virial theorem in terms of loworder density matrices. The most important result is the sum rule (2.14) which represents the identity satisfied by the exchange-correlation force $\boldsymbol{F}_{\text {xc }}(\boldsymbol{r})=-\boldsymbol{\nabla} v_{\text {xc }}(\boldsymbol{r})$ associated with the exact exchange-correlation potential $v_{\text {xc }}(\boldsymbol{r})$. Both objects are functionals of the density $n(\boldsymbol{r})$, which occurs also explicitly in the sum rule. To obtain in Sec. III the sum rules for the exchange and correlation forces separately, Eqs. (3.6b) and (3.7), the adiabatic-connection method also needs to be involved.

It should be mentioned that any of the obtained sum rules for a force can be transformed into an equivalent sum rule for the associated potential. Namely, for $\boldsymbol{F}_{A}(\boldsymbol{r})$ $=-\boldsymbol{\nabla} v_{A}(\boldsymbol{r})$, the equation $\left\langle n \boldsymbol{F}_{A}\right\rangle=\mathbf{0}$ is equivalent to $\left\langle v_{A} \boldsymbol{\nabla} n\right\rangle$ 
$=\mathbf{0}$. For some systems these rules may be satisfied due to symmetry only, e.g., when the density and potential are spherically symmetric.

Evidently, in the continuing search for refined functionals, as motivated in part by critical comments of Wanko et al. ${ }^{1}$ for biological applications, it is important to test how well the exact sum rules, and especially Eq. (2.14) involving the exchange-correlation potential, are obeyed by refined proposals in the future.

\section{ACKNOWLEDGMENTS}

N.H.M. wishes to acknowledge that his contribution to the present article was brought to fruition during visits to the Donastia International Physics Center, San Sebastian, Spain, and to the Institute of Physical Chemistry of PAS, Warsaw, Poland. He especially thanks Professor P. M. Echenique and Professor A. Rubio in San Sebastian and Professor A. Holas in Warsaw for their most generous hospitality. A.R. was partially supported by the EC Sixth Framework Network of Excellence NANOQUANTA (NMP4-CT-2004-500198), the Spanish MCyT, and the Humboldt Foundation under the Bessel research award (2005).

${ }^{1}$ M. Wanko, M. Hoffman, P. Strodet, A. Koslowski, W. Thiel, F. Neese, T. Frauenheim, and M. Elstner, J. Phys. Chem. B 109, 3606 (2005).

${ }^{2}$ A. Holas and N. H. March, Phys. Rev. A 51, 2040 (1995).

${ }^{3}$ N. H. March and W. H. Young, Nucl. Phys. 12, 237 (1959).

${ }^{4}$ M. Levy and J. P. Perdew, Phys. Rev. A 32, 2010 (1985).

${ }^{5}$ D. P. Joubert, J. Chem. Phys. 119, 1916 (2003).

${ }^{6}$ R. G. Parr and W. Yang, Density-Functional Theory of Atoms and Molecules (Oxford University Press, Oxford, 1989).

${ }^{7}$ M. M. Morell, R. G. Parr, and M. Levy, J. Chem. Phys. 62, 549 (1975).

${ }^{8}$ M. Levy and Á. Nagy, Phys. Rev. Lett. 83, 4361 (1999);Á. Nagy and M. Levy, Phys. Rev. A 63, 052502 (2001).

${ }^{9}$ M. Levy and N. H. March, Phys. Rev. A 55, 1885 (1997).

${ }^{10}$ A. Görling and M. Levy, Phys. Rev. B 48, 11638 (1993). 